\title{
Predicting Surgical Factors for Unplanned Overnight Admission in Ambulatory Arthroscopic Surgery of the Knee: A Prospective Cohort in 184 patients
}

\section{Ratthapoom Watcharopas}

Mahidol University

Nadhaporn Saengpetch ( $\nabla$ doctorobb@gmail.com )

Mahidol University

Chusak Kijkunasathian

Mahidol University

Chalermchai Limitlaohaphan

Mahidol University

Chatchawan Lertbutsayanukul

Mahidol University

Chaiyanun Vijittrakarnrung

Mahidol University

Vanlapa Arnuntasupakul

Mahidol University

Lisa Sangkum

Mahidol University

Paphon Sa-ngasoongsong

Mahidol University

\section{Research Article}

Keywords: Unplanned overnight admission, Ambulatory knee arthroscopic surgery, Clinical risk factor, Surgical invasiveness, Tourniquet time

Posted Date: September 14th, 2021

DOI: https://doi.org/10.21203/rs.3.rs-870705/v1

License: (c) (1) This work is licensed under a Creative Commons Attribution 4.0 International License.

Read Full License 


\title{
Predicting Surgical Factors for Unplanned Overnight Admission in Ambulatory Arthroscopic Surgery of the Knee: A Prospective Cohort in 184 patients
}

\section{Author}

Ratthapoom Watcharopas, MD ${ }^{1,3}$ (poom911@hotmail.com)

Nadhaporn Saengpetch, MD1 (doctorobb@gmail.com)

Chusak Kujkunasathian, MD1 (hong3101@gmail.com)

Chalermchai Limitloahaphan, MD' (lerm110@yahoo.com)

Chatchawan Lertbutsayanukul, MD'1 (Ichatchawan@hotmail.com)

Chaiyanun Vijittrakarnrung, MD¹ (drchaiyanun@gmail.com)

Vanlapa Arnuntasupakul, MD² (Vanlapaa@gmail.com)

Lisa Sangkum, MD² (lisa.sangkum@gmail.com)

Paphon Sa-ngasoongsong ${ }^{1}$ (paphonortho@gmail.com)

${ }^{1}$ Department of Orthopedics, ${ }^{2}$ Department of Anesthesia, Faculty of Medicine Ramathibodi Hospital, Mahidol University, Bangkok, Thailand.

${ }^{3}$ Department of Orthopedics, Vejthani Hospital, Bangkok, Thailand.

Corresponding author's information: Assistant Professor Nadhaporn Saengpetch, MD

Address: $\quad$ Department of Orthopedics, Faculty of Medicine Ramathibodi Hospital, Mahidol

University.

270, Rama VI Road, Ratchathewi, Bangkok, Thailand 10400

Tel: +66 2-2011589, Fax: +66 2-201-1599

E-mail address: doctorobb@gmail.com

\begin{abstract}
Background: Unplanned overnight admission (UOA) is an important indicator for quality of care with ambulatory knee arthroscopic surgery (AKAS). However, few studies have explored the factors related to the UOA and how to predict UOA after AKAS. This study aimed to evaluate the effectiveness of a
\end{abstract}


standardized perioperative protocol for the AKAS with UOA and identify whether a correlation exists between the perioperative surgical factors and UOA in the patients undergoing AKAS.

Methods. A prospective cohort study was conducted, between October 2017 and March 2021, in 184 patients. All patients operated on standard AKAS protocol. The UOA was defined as overnight hospitalization of a patient undergoing AKAS. Demographic and perioperative data were recorded, and the procedure was categorized based on the surgical invasiveness based on less invasive $(n=65)$ and more complex surgery $(n=119)$. The clinical risk factors for UOA were identified and analyzed with multivariate logistic regression analysis.

Results. The incidence of UOA in the more complex group (17 cases, 14.3\%) was significantly higher than in the less invasive group ( 3 cases, $4.6 \%)(p=0.049)$, with the incidence of readmission as $0 \%$. The perioperative factors significantly associated with UOA were age, more complex surgery, and tourniquet time $(p<0.10$ all). However, the multivariate regression analysis revealed that tourniquet time was the only significant predictor for UOA (odds ratio $=1.045,95 \%$ confidence interval $=1.022$ to $1.067, p=$ 0.0001 ). The optimal cut-off points of tourniquet time for predicting UOA with the highest Youden index in the less invasive and more complex groups were 56 minutes and 107 minutes, respectively.

Conclusion: The UOA after AKAS is more common in more complex surgery compared to less invasive surgery. Many factors-such as patient factors, surgical invasiveness, and tourniquet time-were also significantly associated with the unplanned admission. However, the results from this study showed that, under strict perioperative management protocol, tourniquet time is the only independent predictor for UOA.

Keywords: Unplanned overnight admission, Ambulatory knee arthroscopic surgery, Clinical risk factor, Surgical invasiveness, Tourniquet time

\section{Background}

Outpatient or ambulatory knee arthroscopic surgery (AKAS) is one of the most common minimally invasive orthopedic surgeries and has been increasingly performed over recent decades ${ }^{1}$ due to the 
numerous advantages (e.g., the treatment cost with unchanged or improved clinical outcomes) compared to inpatient arthroscopic knee surgery ${ }^{2,3}$. However, although AKAS can usually be safely performed as day-case surgery with the improvement of surgical and anesthetic techniques compared to arthroscopic knee surgery, some patients still do not experience the expected postoperative recovery and thus require overnight stay. Previous studies showed that unplanned overnight admission (UOA) ranged from $0.0004 \%$ to $20 \%$, 5, 6 , and the most common cause for UOA was severe postoperative pain ${ }^{6}$. While previous studies mostly focused on the factors associated with readmission after AKAS, especially on anterior cruciate ligament $(A C L)$ reconstruction ${ }^{7,8,9}$, to the best of our knowledge, only a few studies explored the factors related to UOA and how to predict UOA after AKAS ${ }^{10,11}$. Moreover, there were some variations on the perioperative protocol that might affect the UOA after AKAS, such as the anesthetic technique ${ }^{5}$, the use of tourniquet and setting pressure ${ }^{12,13}$, and the standardization of operative procedures ${ }^{4}$. Therefore, this study aimed to evaluate the effectiveness of a standardized perioperative protocol on UOA and identify whether a correlation exists between the perioperative surgical factors and UOA in patients undergoing AKAS.

\section{Materials and Methods}

\section{Study design, participants, inclusion and exclusion criteria}

This study was designed as a single-centered prospective cohort study in a medical university hospital and was approved by our institutional review board (protocol number: ID 09-60-07). Informed consent was obtained from all patients who participated in the study, before the surgery was scheduled, in accordance with the Declaration of Helsinki. The manuscript was prepared according to Strengthening the Reporting of Observational studies in Epidemiology (STROBE) guideline ${ }^{14}$.

The AKAS was developed by a multidisciplinary team comprising sports medicine surgeons, regional anesthesiologists, nurses, and clinical administrators. All patients and their caregivers received preoperative education and counseling for perioperative instructions and postoperative rehabilitation using instructional video and brochures. The study population was patients who underwent AKAS at Ramathibodi Hospital between October 2017 and March 2021. The inclusion criteria were the patients who 1) were aged between 18 and 60 years, 2) could follow perioperative protocol, 3) had American Society of Anesthesiologists (ASA) physical status grade 1 or 2,4 ) had no bleeding disorder, 5) were living around Bangkok or its suburbs, and 6) were willing to participate in this study and give informed consent. The exclusion criteria focused on patients who 1) were unable to communicate due to a physical condition (e.g., hearing loss) or underlying disease (e.g., dementia), 2) had a preoperative diagnosis of radicular pain or neuropathic pain on the operated knee, and 3) refused to participate.

\section{Standard perioperative protocol and criteria for discharge}

All arthroscopic surgeries were randomly performed by six experienced surgeons using the same arthroscopic surgical approach under pneumatic tourniquet with $350 \mathrm{mmHg}$ of pressure. All anesthesia 
was administered under the care of two anesthesiologists (VA and LS) using the same protocol as combined ultrasound-guided adductor canal block (ACB) and general anesthesia. All patients were first sedated with $0.03 \mathrm{mg} / \mathrm{kg}$ of midazolam and $0.5-1.0 \mathrm{mcg} / \mathrm{kg}$ of fentanyl before the anesthetic procedure. The local anesthetic for ACB was $15-20 \mathrm{~mL}$ of $0.5 \%$ bupivacaine. Standardized general anesthesia was initiated with 1-2 mg/kg of propofol and 50-100 mcg of fentanyl intravenously with or without muscle relaxant. Then, a laryngeal mask airway or endotracheal tube was inserted, and anesthesia was maintained with 0.8-1.0 MAC of sevoflurane or desflurane using an oxygen/air mixture. During operation, additional intravenous $0.1-0.2 \mathrm{mg} / \mathrm{kg}$ of morphine (maximum $10 \mathrm{mg}$ ), 5-10 mg dexamethasone, $30 \mathrm{mg}$ ketorolac or $40 \mathrm{mg}$ parecoxib, and $4-8 \mathrm{mg}$ of ondansetron were given.

After the completed operation, all patients were subsequently transferred to the postanesthetic care unit (PACU) for at least 1 hour until showing a modified Aldrete score of $\geq 9^{15}$; patients were then transferred to a day surgery unit. Additional analgesics (2-4 mg intravenous morphine and $0.5-1.0 \mathrm{~g}$ oral acetaminophen) and antiemetics (intravenous 2-4 mg ondansetron or 5-10 mg metoclopramide) were given if the patients had a 10-point numeric rating scale (NRS) of $\geq 4$ or postoperative nausea or vomiting (PONV), respectively. All patients were assessed by one of the authors (RW) at 6 hours after surgery for appropriate discharge. The discharge criteria were as follows: 1) NRS $\leq 3,2$ ) no PONV, 3) able to independently walk with crutches, 4) no urinary retention, 5) no abnormal surgical wound bleeding, and 6) no reoperation required for any cause. The patients who did not meet the discharge criteria were admitted into the orthopedic ward for further treatment. All patients were followed at 24 and 48 hours postoperatively by telephone, and at 2 weeks, 6 weeks, and 3 months after surgery at the sports medicine outpatient clinic or via a telephone interview for clinical evaluation.

\section{Data collection and outcome measurement}

Demographic data included the following: age, gender, body mass index (BMI), ASA physical status, diagnosis, and preoperative international knee documentation committee (IKDC) score. The perioperative and postoperative data-such as operation, surgical category and tourniquet time, discharge status, incidence of UOA, and cause of UOA-were recorded. The surgical category was defined as either a less invasive and more complex surgical procedure, based on the surgical invasiveness of outpatient knee surgery, following the prior study by Williams et al. ${ }^{5,16}$ (Table 1). The cause of UOA was defined as any patient problems that did not meet the discharge criteria.

\section{Statistical analysis}

STATA SE version 16.0 (StataCorp, College Station, Texas, USA) was used to analyze data. Categorical data were analyzed by the Fisher Exact test or chi-square test, and continuous variables were analyzed using unpaired t-test or Wilcoxon rank-sum test for data as appropriate. Univariate logistic regression analysis was used to determine the predictors associated with UOA. Multivariate logistic 
regression analysis with stepwise was performed and included variables with a $p$-value less than 0.10 from the univariate analysis. Significant difference was defined as $p$-value $<0.05$. The Spearman coefficient and scatter plot were used to identify the correlation between the significant variable and the most common cause of unplanned admission. The receiver operating characteristic $(R O C)$ curve with the Youden index was used to determine the cut-off value for the significant variable. The performance test of the prediction model was calculated to determine the sensitivity, specificity, and positive and negative predictive value in each model.

\section{Results}

\section{Demographic data}

During the 2.5-year study period (between October 2017 and March 2021), a total of 184 patients who underwent AKAS were enrolled consecutively into this study (Fig. 1). Demographic data are shown in Table 2. Among these patients, the average patient age was 33.4 years (range 15-62 years), and 143 patients $(77.7 \%)$ were male. The average BMI and preoperative IKDC score were $25.1 \mathrm{~kg} / \mathrm{m}^{2}$ (range $16.6-34.6 \mathrm{~kg} / \mathrm{m}^{2}$ ) and 52.1 (range 24-97), respectively. The operations were categorized as either less invasive (65 cases, 35.3\%) and more complex surgery (119 cases, 64.7\%). The less invasive group comprised meniscus-alone surgery (51 cases) and other mild surgeries, (14 cases); mild surgeries included arthroscopic debridement with or without loose bodies removal (8 cases), plica resection (2 cases), excision pigmented villonodular synovitis (2 cases), and implant removal (2 cases). The more complex group comprised ACL reconstruction alone (28 cases), ACL reconstruction with meniscal surgery (90 cases), and medial patellofemoral ligament reconstruction with lateral retinacular release (1 case). The median tourniquet time was 93 minutes (range 20-163 minutes). After AKAS, 20 cases (10.9\%) were admitted unexpectedly due to NRS $\geq 4$ (17 cases), PONV ( 1 case), NRS $\geq 4$ and severe dizziness ( 1 case), and need of reoperation due to unexpected retained and broken metal guide (1 case). There were no readmissions during the 3-month postoperative follow-up period.

Table 3 shows the data comparison between the patients who had been treated with less invasive surgery $(n=65)$ and more complex surgery $(n=119)$. The incidence of UOA in the more complex group (17 cases, $14.3 \%$ ) was significantly higher than among those in the less invasive group (3 cases, $4.6 \%$ ) $(p=0.049)$. The more complex group also showed a significantly higher age and male proportion, lower $\mathrm{BMI}$ and preoperative IKDC, and longer tourniquet time, compared to the less invasive group $(p<0.01$ all).

Risk factors for unplanned admission and correlation with the most common cause of admission Table 4 demonstrates the univariate and multivariate logistic regression analysis for UOA. Based on univariate analysis, the perioperative factors significantly associated with UOA were age, more complex surgery, and tourniquet time $(p<0.10$ all). However, multivariate regression analysis revealed that 
tourniquet time was the only significant predictor for UOA (odds ratio $[O R]=1.045,95 \%$ confidence interval $[\mathrm{Cl}]=1.022$ to $1.067, \mathrm{p}=0.0001)$. The area under the curve of this prediction model was 0.798 $(95 \% \mathrm{Cl}=0.733$ to 0.854$)$. The Spearman coefficient and scatter plot showed fair correlation ${ }^{17}$ between NRS score at 6 hours and tourniquet time $\left(r_{s}=0.364, p<0.0001\right)$ (Fig. 1).

\section{ROC analysis}

The results of using ROC analysis to predict the UOA using tourniquet time are detailed in Table 5. The optimal cut-off point of tourniquet time from this prospective cohort study $(n=184)$ with the highest Youden index was 103 minutes (Youden index $=0.476$, sensitivity $75.0 \%$, specificity $72.6 \%$, PPV 25.0\%, and NPV 96.0\%). Regarding the surgical invasiveness, for those in the less invasive group $(n=65)$, the optimal cut-off point for the tourniquet time with the highest Youden index was 56 minutes (Youden index $=0.726$, sensitivity $100.0 \%$, specificity $72.6 \%$, PPV 15.0\%, and NPV 100.0\%). For the more complex surgery $(n=119)$, the optimal cut-off point for the tourniquet time with the highest Youden index was 107 minutes (Youden index $=0.422$, sensitivity $76.5 \%$, specificity $65.7 \%$, PPV 27.1\%, and NPV 94.4\%).

\section{Discussion}

The results from this study showed that the incidence of UOA from AKAS was as high as $10.9 \%$ (4.6\% in less invasive group and $14.3 \%$ in more complex group) with the incidence of readmission at $0 \%$ and postoperative pain as the most common UOA cause, comparable to the results from previous studies $^{4,5}$ (Table 2). In addition, the present study demonstrated a significant difference in the characteristics of patients who underwent the AKAS based on the invasiveness of the operations (Table 3), which was also comparable with the results in the previous study by Saltzman et al. where there was significant difference in the demographic data (e.g., age, gender, and BMI) between the patients who received different arthroscopic surgery ${ }^{18}$. Our results also showed that the patients' characteristics in more complex group were significantly higher in age and proportion of male gender, higher in ASA physical status, longer in tourniquet time, and greater in UOA, compared to those in the less invasive group. These results are comparable with the previous study by Williams et al. as the UOA in the more complex surgery group was significantly higher than for those who underwent less invasive surgery ${ }^{5}$.

Regarding the multivariate analysis, our results showed that tourniquet time was a significant independent predictor for the UOA in this study, which was compatible with the results from previous studies (Table 4). The prediction model also demonstrated that the cut-off points for tourniquet time in the whole cohort, less invasive group, and more complex group (103 minutes, 56 minutes, and 107 minutes, respectively) could predict the UOA with a sensitivity of $75.0 \%, 100.0 \%$, and $76.5 \%$, respectively. These findings imply that longer tourniquet time increases the risk of severe postoperative pain and the risk of UOA. Such implications align with results in previous studies by Lutz et al ${ }^{19}$ and Boddapati et $\mathrm{al}^{10}$ that prolonged tourniquet time of more than 50 minutes was one risk factor of severe postoperative pain (NRS 
$\geq 7)^{19}$ and that procedures longer than 90 minutes are a risk factor for $\mathrm{UOA}^{10}$. Moreover, the present study revealed that the cut-off point for the AKAS procedure could vary between less invasive and more complex surgery. Therefore, the cut-off time for these different procedures should be based on the level of surgical invasiveness.

Certain limitations in the present study should be acknowledged. First, although this study was designed as a prospective observational study with strict inclusion and exclusion criteria, and standardization perioperative protocol, the sample size in our study was relatively small due to the COVID-19 situation. However, our results could demonstrate the significant effect of surgical invasiveness in patients undergoing AKAS (Table 3) and potentially reveal the statistically significant independent predictor of UOA by multivariate analysis (Table 4). Second, the incidence of UOA and the findings in this study might be affected by the study criteria. Due to the inclusion criteria of ASA status grade 1 and 2 and the absence of a bleeding disorder, some potential factors for UOA (e.g., chronic disease, morbid obesity, and smoking status ${ }^{10,11,20}$ ) would be significantly lower or not found in our study population. Lastly, although this study showed that tourniquet time significantly predicted the UOA, our results may not be directly applicable in the other conditions related to the use of tourniquet, such as arthroscopic knee surgery without tourniquet inflation ${ }^{13}$ and the setting of tourniquet pressure based on limb occlusion pressure (this study used only a $350-\mathrm{mmHg}$ tourniquet pressure setting protocol) ${ }^{12}$. Therefore, future studies should employ a larger sample size, and multicentered prospective studies with different surgical protocol are still required to explore the other predicting factors and benefits of the tourniquet setting protocol.

\section{Conclusion}

This study showed that the incidence of UOA after AKAS could be as high as $10.9 \%$ and that the most common cause of UOA was pain-related. Moreover, many factors-such as patient factors, surgical invasiveness, and tourniquet time-were significantly associated with unplanned admissions. However, tourniquet time was the only independent predictor for UOA with the cut-off point of 107 minutes.

\section{Declarations}

\section{1 abbreviations}

ACB: Adductor canal block

ACL: Anterior cruciate ligament

AKAS: Ambulatory knee arthroscopic surgery

BMI: Body mass index

$\mathrm{Cl}$ : Confidence interval

IKDC: International knee documentation committee 
NPV: Negative predictive value

NRS: Numeric rating scale

OR: Odds ratio

PONV: Postoperative nausea or vomiting

PPV: Positive predictive value

ROC: Receiver operating characteristic

UOA: Unplanned overnight admission

6.2 Ethics approval and consent to participate. This study was approved by the Ethical Clearance Committee on Human Rights Related to Research Involving Human Subjects, Faculty of Medicine Ramathibodi Hospital, Mahidol University (protocol number: ID 09-60-07). All patients were appropriately informed and gave consent before participating in this study, in accordance with the Declaration of Helsinki.

6.3 Consent for publication. Not applicable

6.4 Availability of data and material. The datasets generated and/or analyzed during the current study are available from the corresponding author upon any reasonable request.

6.5 Competing interests. All authors declare that they have no conflict of interest.

6.6 Funding. This study was funded by the Faculty of Medicine Ramathibodi Hospital, Mahidol University.

6.7 Authors' contributions. Nadhaporn Saengpetch and Ratthapoom Watcharopas are the main researchers who designed and performed the study and prepared the manuscript.

Chatchawan Lertbutsayanukul and Chaiyanun Vittrakarnrung are orthopedic sports medicine surgeons who assisted in data collection.

Vanlapa Arnuntasupakul and Lisa Sangkum are experienced anesthesiologists who performed all anesthesia.

Paphon Sa-ngasoongsong is a senior orthopedic surgeon who assisted in manuscript preparation. Chusak Kijkunasathian and Chalermchai Limitlaohaphan are senior orthopedic consultants who assisted in the research process.

6.8 Acknowledgements. The authors wish to thank Department of Orthopedics, Faculty of Medicine Ramathibodi Hospital, Mahidol University for the kind assistance and permission to carry out this study.

\section{References}


1. Kim S, Bosque J, Meehan JP, Jamali A, Marder R. Increase in outpatient knee arthroscopy in the United States: a comparison of National Surveys of Ambulatory Surgery, 1996 and 2006. J Bone Joint Surg Am. 2011;93(11):994-1000.

2. Robinson JC, Brown TT, Whaley C, Bozic KJ. Consumer choice between hospital-based and freestanding facilities for arthroscopy: impact on prices, spending, and surgical complications. J Bone Joint Surg Am. 2015;97(18):1473-81.

3. Ferrari D, Lopes TJA, Franca PFA, Azevedo FM, Pappas E. Outpatient versus inpatient anterior cruciate ligament reconstruction: a systematic review with meta-analysis. Knee. 2017;24(2):197-206.

4. Khan T, Jackson WF, Beard DJ, Marfin A, Ahmad M, Spacie R, et al. The use of standard operating procedures in day case anterior cruciate ligament reconstruction. Knee. 2012;19(4):464-8.

5. Williams BA, Kentor ML, Vogt MT, Williams JP, Chelly JE, Valalik S, et al. Femoral-sciatic nerve blocks for complex outpatient knee surgery are associated with less postoperative pain before same-day discharge: a review of 1,200 consecutive cases from the period 1996-1999. Anesthesiology. 2003;98(5):1206-13.

6. Goldfarb CA, Bansal A, Brophy RH. Ambulatory surgical centers: a review of complications and adverse events. J Am Acad Orthop Surg. 2017;25(1):12-22.

7. Bokshan SL, DeFroda SF, Owens BD. Risk factors for hospital admission after anterior cruciate ligament reconstruction. Arthroscopy. 2017;33(7):1405-11.

8. Cooper JD, Lorenzana DJ, Heckmann N, McKnight B, Mostofi A, Gamradt SC, et al. The effect of obesity on operative times and 30-day readmissions after anterior cruciate ligament reconstruction. Arthroscopy. 2019;35(1):121-9.

9. Lu Y, Lavoie-Gagne O, Khazi Z, Patel BH, Mascarenhas R, Forsythe B. Inpatient admission following anterior cruciate ligament reconstruction is associated with higher postoperative complications. Knee Surg Sports Traumatol Arthrosc. 2020;28(8):2486-93.

10. Boddapati V, Fu MC, Nwachukwu BU, Camp CL, Spiker AM, Williams RJ, et al. Procedure length is independently associated with overnight hospital stay and 30-day readmission following anterior cruciate ligament reconstruction. Knee Surg Sports Traumatol Arthrosc. 2020;28(2):432-8.

11. Min CJ, Partan MJ, Koutsogiannis P, Iturriaga CR, Katsigiorgis G, Cohn RM. Risk factors for hospital admission in patients undergoing outpatient anterior cruciate ligament reconstruction: a national database study. J Orthop. 2020;22:436-41.

12. Masri BA, Eisen A, Duncan CP, McEwen JA. Tourniquet-induced nerve compression injuries are caused by high pressure levels and gradients - a review of the evidence to guide safe surgical, prehospital and blood flow restriction usage. BMC Biomed Eng. 2020;2:7.

13. Reda W, EIGuindy AMF, Zahry G, Faggal MS, Karim MA. Anterior cruciate ligament reconstruction; is a tourniquet necessary? A randomized controlled trial. Knee Surg Sports Traumatol Arthrosc. 2016;24(9):2948-52. 
14. von Elm E, Altman DG, Eggger M, Pacock SJ, Gotzshe PC, Vandenbroucke JP. The Strengthening the Reporting of Observational Studies in Epidemiology (STROBE) statement guidelines for reporting observational studies. Ann Intern Med 2007;147:573-7.

15. Aldrete JA. The post-anesthesia recovery score revisited. J Clin Anesth. 1995;7(1):89-91.

16. Williams BA, Kentor ML, Williams JP, Vogt MT, DaPos SV, Harner CD, et al. PACU bypass after outpatient knee surgery is associated with fewer unplanned hospital admissions but more phase II nursing interventions. Anesthesiology. 2002;97(4):981-8.

17. Akoglu H. User's guide to correlation coefficients. Turk J Emerg Med. 2018;18(3):91-3.

18. Saltzman BM, Cvetanovich GL, Bohl DD, Cole BJ, Bach BR, Jr., Romeo AA. Comparisons of patient demographics in prospective sports, shoulder, and national database initiatives. Orthop J Sports Med. 2016;4(9):2325967116665589.

19. Lutz C, Baverel L, Colombet P, Cournapeau J, Dalmay F, Lefevre N, et al. Pain after out-patient vs. in-patient ACL reconstruction: French prospective study of 1076 patients. Orthop Traumatol Surg Res. 2016;102(8S):S265-S70.

20. Padwal JA, Burton BN, Fiallo AA, Swisher MW, Gabriel RA. The association of neuraxial versus general anesthesia with inpatient admission following arthroscopic knee surgery. J Clin Anesth.

2019;56:145-50. 
Figures

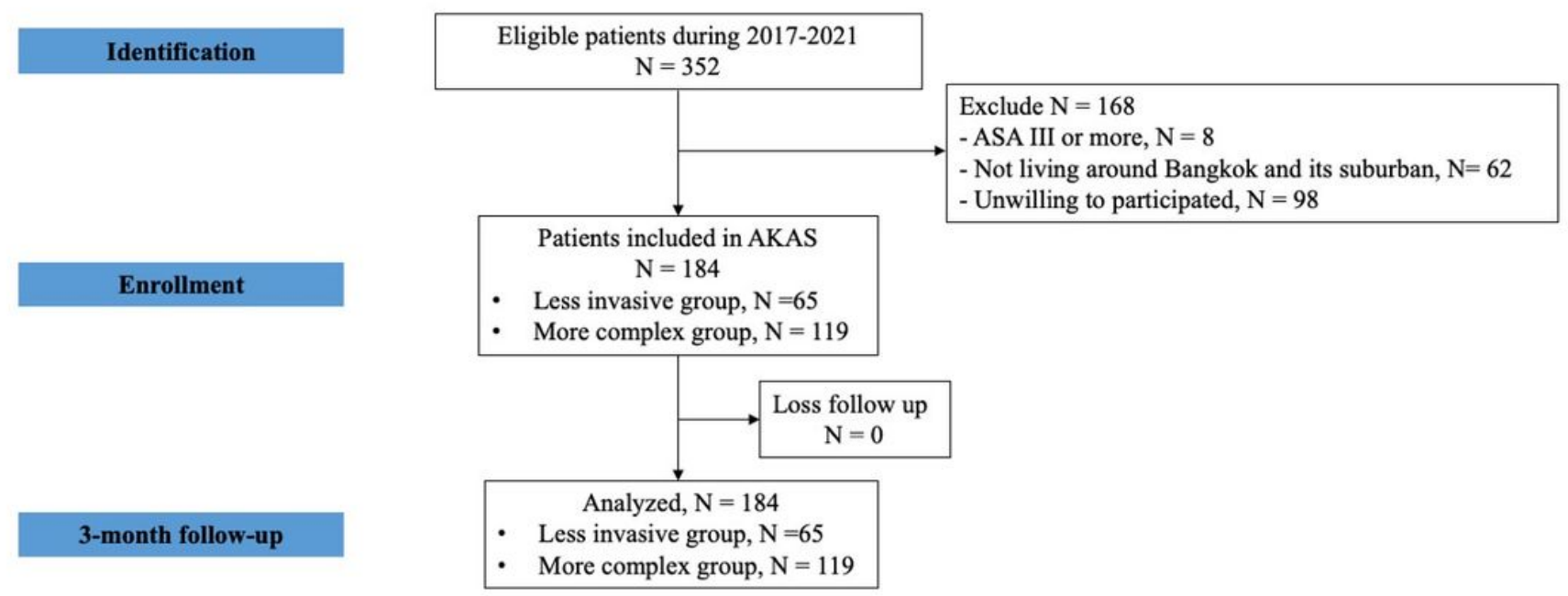

Figure 1

Flow diagram of this study. AKAS ambulatory knee arthroscopic surgery 